\title{
RESOLUTION OF POSITIVE OPERATORS
}

\author{
BY R. V. $\mathrm{CHACON}^{1}$
}

Communicated by G. A. Hedlund, June 18, 1962

We begin with a $\sigma$-finite measure space $(S, \mathcal{F}, \mu)$ and a positive linear operator $T$ on $L_{1}$ with $\|T\|_{1} \leqq 1$. The main result of this note is the following theorem:

Theorem 1. If $p \in L_{1}$ and is non-negative and if $T$ is regular, then

$$
L_{1} \stackrel{p}{=} I_{p} \oplus \bar{M}^{2}
$$

where

$$
\begin{aligned}
& M=\left\{g: g=f-T f, f \in L_{1}\right\}, \\
& I_{p}=\left\{h p: T(h p)=h T p, h p \in L_{1}\right\} .
\end{aligned}
$$

Before proceeding we give some definitions, in particular that of a regular operator. We have used this term in the statement of Theorem 1.

Definition. $T$ is regular if

$$
\sum_{k=0}^{\infty} T^{k} p(s)=+\infty
$$

whenever $p \in L_{1}$ is strictly positive.

We summarize results [2], reducing general operators to regular operators in the following theorem:

Theorem 2. There exists a set $S_{1}$ so that the restriction of $T$ to $L_{1}\left(S_{1}, \mathscr{F}_{1}, \mu\right)$, where $\mathfrak{F}_{1}=\mathfrak{F} \cap S_{1}$, is regular and so that $\sum_{k=0}^{\infty}\left|T^{k} f\right|<+\infty$ on $c S_{1}$. Furthermore, for each $f \in L_{1}\left(S_{1}, \mathfrak{F}_{1}, \mu\right)$ there exists a $f_{S_{1}} \in$ $L_{1}\left(S_{1}, \mathscr{F}_{1}, \mu\right)$ so that

$$
\lim _{n \rightarrow \infty} \frac{\sum_{k=0}^{n} T^{k}\left(f-f_{S_{1}}\right)}{\sum_{k=0}^{n} T^{k} p}=0
$$

on $S_{1} \cap\left\{s: \sum_{k=0}^{\infty} T^{k} p(s)>0\right\}$.

${ }_{1}^{1}$ The work reported in this paper was carried out under a grant from the National Science Foundation, G19046.

${ }^{2} A={ }^{p} B$ if the spaces are equal with respect to the seminorm obtained by integration over the support of $\sum_{k=0}^{\infty} T^{k} p(s)$. 
Since $L_{1}^{*}$ is isometric to $L_{\infty}$, we regard $T^{*}$, the adjoint of $T$, as a linear operator on $L_{\infty}$. It follows that $T^{*}$ is positive and that $\left\|T^{*}\right\|_{\infty} \leqq 1$.

DEFINITION. Let $I_{\infty}$ be the space of functions of $L_{\infty}$ which are invariant under $T^{*}$, let $\mathfrak{g}$ be the Borel field generated by the functions of $I_{\infty}$ and let $I_{M}$ be the class of functions which are measurable with respect to $\mathfrak{I}$ ( $I_{M}$ is not assumed to be a subspace of $L_{\infty}$ or $L_{1}$ ).

THEOREM 3. If $p \in L_{1}$ and is non-negative and if $T$ is regular, then

$$
\left\{h p: T(h p)=h T p, h p \in L_{1}\right\}=\left\{h p: h p \in L_{1}, h \in I_{M}\right\} \text {. }
$$

Theorems 1 and 3 can be combined to yield the following result:

TheOREM 4. If $p \in L_{1}$ and is non-negative and if $T$ is regular, then

$$
L_{1} \stackrel{p}{=} I_{p} \oplus \bar{M}
$$

where

$$
\begin{aligned}
& M=\left\{g: g=f-T f, f \in L_{1}\right\}, \\
& I_{p}=\left\{h p: h p \in L_{1} \text { and } T^{*} h=h\right\}
\end{aligned}
$$

and $T^{*} h=h$ provided the domain of definition of $T^{*}$ is extended in a natural way.

We note that this result is analogous to the Kakutani-Yosida mean ergodic theorem in which a similar resolution is obtained for operators on reflexive Banach spaces. This analogy is more apparent if the reflexive Banach space is Hilbert space and we state their theorem specialized to the Von Neumann case:

MeAN ERgodic Theorem. If $H$ is Hilbert space and if $T$ is a linear transformation on $H$ with $\|T\| \leqq 1$ then

$$
H=I \oplus \bar{M}
$$

where

$$
\begin{aligned}
M & =\{g: g=f-T f, f \in H\}, \\
I & =\left\{f: T^{*} f=f\right\} .
\end{aligned}
$$

Since the fixed points of a contraction in Hilbert space are the same as those of its adjoint, $I=\{f: T f=f\}$.

Theorem 1 can be used to obtain the results of [ 1 ] and [3], avoiding the somewhat complicated procedure if [1] which involves showing that the integral of a function is infinite if the limit of the ratio in question fails to exist. We have, in a forthcoming paper, also obtained some 
results for operators not assumed positive by means of a reduction procedure [4] and the present theorems.

We are indebted to the work of Eberhard Hopf for suggesting that a resolution of this type is possible.

\title{
BibLIOGRAPHY
}

1. R. V. Chacon and D. S. Ornstein, $A$ general ergodic theorem, Illinois J. Math. 4 (1960), 153-160.

2. R. V. Chacon, The influence of the dissipative part of a general Markov process, Proc. Amer. Math. Soc. 11 (1960), 957-961.

3. - Identification of the limit of operator averages, J. Math. Mech. (to appear).

4. - Convergence of operator averages, (to appear).

BROWN UNIVERSITY

\section{TCHEBYCHEFF QUADRATURE IS POSSIBLE ON THE INFINITE INTERVAL ${ }^{1}$}

\author{
BY J. L. ULLMAN
}

Communicated by J. L. Doob, May 29, 1962

The purpose of this announcement is to state a theorem on Tchebycheff quadrature which answers a question posed in [1], and to discuss the proof. Complete details will appear elsewhere.

\section{Tchebycheff quadrature.}

Definition 1.1. A unit mass distribution on $(-\infty, \infty)$ possessing moments of all positive integer order will be said to belong to class $D$.

Definition 1.2. Let $\psi$ be an element of $D$ and $n$ a positive integer. We refer to the equations

$$
\frac{1}{n} \sum_{i=1}^{n} x_{i, n}^{k}=\int x^{k} d \psi, \quad k=1, \cdots, n
$$

as the equations $(\psi, n)$. These equations admit a solution $x_{1, n}, \cdots$, $x_{n, n}$ which is unique up to permutation of the first index.

Definition 1.3. $T$ quadrature is said to be possible for an element $\psi$ of $D$ if equations $(\psi, n)$ have real solutions for every positive integer $n$. If $T$ quadrature is possible for $\psi$ it is called a $T$ distribution.

1 This research was supported in part by National Science Foundation Grant No. G19654. 